\title{
Umidade da madeira como fator de influência no processamento
}

\author{
Anna Carolina de Almeida Andrade 1 , Roqueline Lins Santos ${ }^{1}$, Cilene dos Santos, Alessandra Dantas \\ Fonseca $^{1}$, Antônio Mendes de Santana Neto ${ }^{1}$, Antônio Américo Cardoso Júnior ${ }^{1}$
}

\begin{abstract}
RESUMO: Características físicas da madeira, como a umidade, influenciam diretamente na qualidade da superfície, resistência ao corte e energia requerida para o processamento. Portanto, o objetivo foi conhecer a relação do gasto de energia, para realizar o corte transversal, com a umidade da madeira de nove espécies. Foram utilizados cinco corpos de prova por espécie para coleta de dados de energia específica de corte. Os corpos de prova foram processados transversalmente em serra circular de carrinho. A velocidade de avanço foi mantida constante pelo alimentador de avanço mecânico e a velocidade de corte foi fixada durante todo o processo. Assim, não exerceram fonte de variação nos dados de energia específica de corte. Posteriormente, foram calculados os valores de energia específica, considerando o volume de madeira removida, para cada espécie. Os resultados mostraram que houve relação mediana entre os dados de energia específica de corte e umidade para os cortes transversal $(r=-0,462)$. A relação obtida entre a energia específica de corte e a umidade da madeira foi inversamente proporcional, ou seja, maiores umidades facilitam o corte da madeira. Concluise, que a umidade exerce de fato influência no corte, afetando diretamente no quanto a madeira vai requerer de esforço durante seu processamento mecânico.
\end{abstract}

Palavras-chave: usinagem, energia específica de corte, propriedade física da madeira.

\section{Moisture of wood as a factor influencing processing}

\begin{abstract}
Physical characteristics of wood, such as moisture, directly influence the surface quality, cutting strength and energy required for processing. Therefore, the aim was to know the relation of the energy spent, to carry out the transversal cut, with the wood moisture of nine species. Five specimens per specie were used for data collection of specific cutting energy. The specimens were processed transversely in a circular cart saw. The feed rate was kept constant by the feed feeder and the cutting speed was set during the entire process. Thus, they did not exercise a source of variation in the specific energy data of cut. Subsequently, the specific energy values were calculated, considering the volume of wood removed, for each species. The results showed that there was a median relationship between the specific energy data of cut and moisture for the cross sections $(\mathrm{r}=-0.462)$. The relationship obtained between the specific cutting energy and the moisture of the wood was inversely proportional, that is, higher moisture facilitates the cutting of the wood. It is concluded that the moisture exerts influence in the cut, directly affecting how much the wood will require of effort during its mechanical processing.
\end{abstract}

Keywords: wood workability, specific cutting energy, physical property of wood.

\section{INTRODUÇÃO}

Informações sobre o comportamento da madeira durante a usinagem é uma necessidade ligada ao processamento dentro de indústrias madeireiras, principalmente por razões econômicas e produtivas. Máquinas e ferramentas fabricadas para o processamento de madeira, bem como os operadores, precisam de informações confiáveis ser sobre os principais fatores que influenciam no processo de usinagem (EYMA et al., 2004).

Neste contexto, a avaliação da energia específica de corte é muito importante para garantir que as máquinas sejam eficientes no processamento mecânico, sem desperdícios energéticos. Isso porque, os principais problemas enfrentados pela indústria madeireira, geralmente estão vinculados aos altos gastos com energia e as constantes paradas de produção devido à ultrapassagem dos limites de potência nominal dos motores elétricos (ANDRADE et al., 2018).

A umidade da madeira é uma das principais características que influenciam no corte. Contudo, há escassez de estudos que mensurem em que magnitude essas propriedades interferem na energia que é requerida durante o processamento. Em geral, os estudos que se concentraram na interação entre a umidade da madeira e as forças envolvidas no processo de corte, relatam que há tendência descendente das forças de corte devido ao aumento

\footnotetext{
Recebido em 05/10/2019, Aceito para publicação em 15/10/2019

${ }^{1}$ Universidade Federal de Sergipe

*e-mail: carol_bertges@hotmail.com
} 
da umidade da madeira (FRANZ, 1958; KOCH, 1964; LUČIĆ et al., 2004; MORADPOUR et al., 2013). No entanto, as variações neste comportamento podem ocorrer dependendo das espécies e da faixa de umidade utilizadas (LOEHNERTZ, COOZ, 1998).

Visto isto, objetivou-se conhecer a relação do gasto de energia, para realizar o corte transversal, com a umidade da madeira de nove espécies.

\section{MATERIAL E MÉTODOS}

Utilizaram-se madeiras de nove espécies com o intuito de garantir variação das umidades a fim de relacioná-las à energia específica de corte. As nove espécies e suas respectivas médias das umidades encontram-se expostas na Tabela 1.

Tabela 1. Valores médios de umidade base seca para as nove espécies avaliadas.

\begin{tabular}{lc}
\hline \multicolumn{1}{c}{ Espécies } & Umidade Base Seca (\%) \\
\hline Toona ciliata & 16,7 \\
Cryptomeria japônica & 19,8 \\
Tectona grandis & 15,8 \\
Eycalyptus grandis & 17,8 \\
Qualea dinizii & 15,9 \\
E. saligna & 19,4 \\
Bowdichia nitida & 13,0 \\
Hymenaea spp & 14,7 \\
Tabebuia serratifolia & 12,4 \\
\hline
\end{tabular}

Para a análise de energia específica de corte foram utilizados cinco corpos de prova por espécie. As dimensões dos corpos de prova foram variadas para garantir as maiores dimensões possíveis. Para tanto, os corpos de prova foram preparados para os ensaios passando por desempeno, desengrosso e apara dos topos.

Posteriormente ao preparo, os corpos de prova foram processados, de modo aleatório, na serra circular de carrinho. A usinagem dos corpos de prova foi aleatória para distribuir entre as nove espécies (45 corpos de prova) o efeito do desgaste do dente da ferramenta, para que dessa forma a condição de afiação da ferramenta não fosse fonte de variação nos dados de energia específica de corte.

A máquina utilizada era dotada de sistema de exaustão, alimentador de avanço mecânico acoplado ao inversor de frequência (CFW 06). Desse modo, tanto a velocidade de avanço $\left(8,5 \mathrm{~m}^{*} \mathrm{~min}^{-1}\right)$ como a de corte $\left(55 \mathrm{~m}^{*} \mathrm{~s}^{-1}\right)$ foram mantidas constantes, de modo a não exercerem influência na variação dos valores de energia específica de corte. Utilizou-se serra nova com 36 dentes e $300 \mathrm{~mm}$ de diâmetro para que a ferramenta não fosse também fonte de variação nos dados.
Foram executados seis cortes transversais (família $0^{\circ}-90^{\circ}$ ) consecutivos em cada corpo de prova para cada espécie. Ao final dos seis cortes transversais para cada corpo de prova das nove classes de densidade foram retirados corpos de prova para medição da umidade conforme descrição da norma NBR 7190 (ABNT, 1997) e ilustrado na Figura 1.

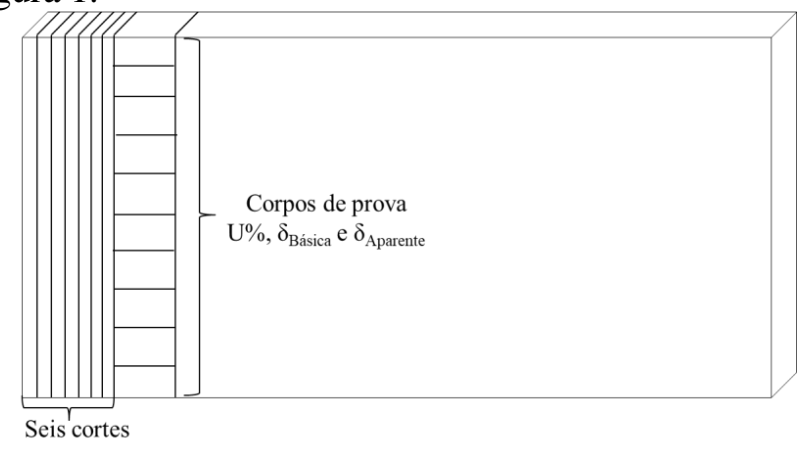

Figura 1 - Esquema da realização dos ensaios, evidenciando os seis cortes transversais e posição de retirada dos corpos de prova para a análise da umidade da madeira.

Por meio de inversor de frequência (CFW 08) acoplado ao motor de acionamento do eixo porta ferramentas, foi possível monitorar os cortes transversais na serra circular de carrinho. Os dados gerados pelo inversor de frequência são os dados de torque mecânico e a rotação do eixo porta ferramentas, esses dados são fornecidos durante toda a coleta de dados.

Os dados adquiridos pelo inversor de frequência foram exportados para o Excel onde foram realizados os ajustes e confecção dos gráficosrespostas. A captura dos dados foi realizada pelo software de parametrização sem interrupções ao longo do tempo e foram adotadas quatro leituras por segundo. Isto resultou em uma extensa base de dados com valores de porcentagem de torque para momentos ociosos, em que não se realizou o corte efetivo, e momentos a cheio, que é quando o motor está trabalhando com carga para realizar o corte. Desse modo houve necessidade de filtrar a base de dados geral (FIGURA 2), conforme descrito por Braga (2011). 


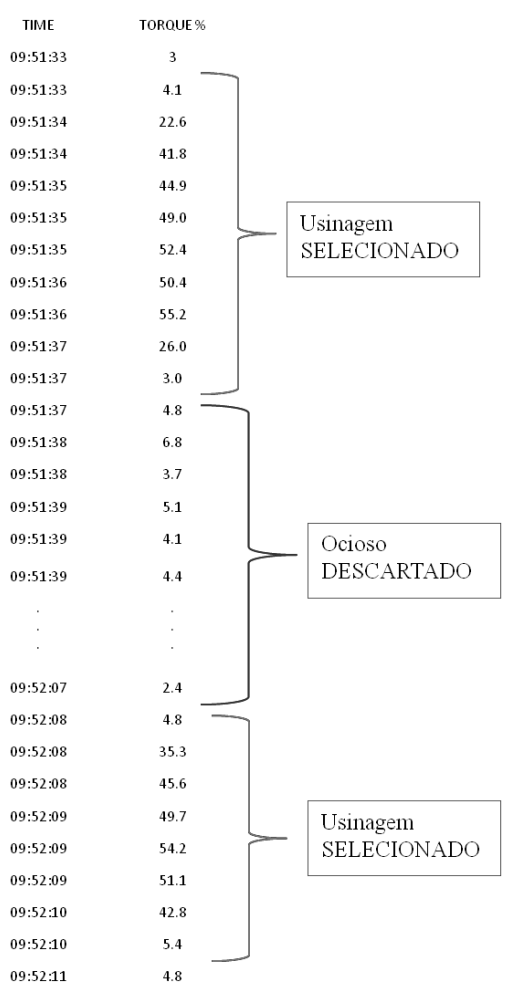

Figura 2 - Esquema da realização do ajuste dos dados em que ocorre o descarte dos valores ociosos, em que não houve corte da madeira.

Posteriormente ao procedimento de ajuste da base de dados procedeu-se conforme metodologia proposta por Souza (2009) em que se utilizou sequencia de equações matemáticas para chegar aos valores de energia específica de corte conforme demostrados pelas Equações 1, 2 e 3.

$$
\text { Potencial }=\frac{T_{m e c} \times n \times 0,0014 \times 736}{1000} \quad(\text { Equação } 1)
$$

em que: Potência $=$ potência de corte $(\mathrm{kw}) ; \mathrm{T}=$ torque mecânico do motor $(\mathrm{kgf} * \mathrm{~m}) ; \mathrm{n}=$ rotação $\left(\min ^{-1}\right)$.

$$
E=\frac{\text { PotênciaxC }}{V f} \times 60 \text { (Equação 2) }
$$

em que: $\mathrm{E}=$ energia $(\mathrm{kj}) ;$ Potência $=$ potência de corte $(\mathrm{kw}) ; \mathrm{C}=$ comprimento do corte $(\mathrm{m}) ; V_{f}=$ velocidade de avanço $\left(\mathrm{m}^{*} \min ^{-1}\right)$

$$
E s=\frac{E}{c \times e \times K} \quad(\text { Equação 3) }
$$

em que: $\mathrm{Es}=$ energia específica $\left(\mathrm{kj}^{*} \mathrm{~cm}^{-3}\right) ; \mathrm{E}=$ energia $(\mathrm{kj}) ; \mathrm{c}=$ comprimento da peça $(\mathrm{cm}) ; \mathrm{e}=$ espessura da peça $(\mathrm{cm}) ; \mathrm{K}=$ espessura do dente da ferramenta $(\mathrm{cm})$.

$\mathrm{Na}$ avaliação do experimento foi utilizado o delineamento inteiramente casualizado, considerando como fator de variação as nove espécies. Foram realizadas cinco repetições para cada espécie, totalizando 45 repetições para os dados de energia específica de corte.

A análise de variância (ANOVA) foi realizada à $5 \%$ de significância, essa sendo significativa foi realizado o teste de Scott-Knott à $5 \%$ de significância (Scott \& Knott, 1974). Na verificação dos pressupostos da ANOVA foi realizado o teste de Shapiro- Wilk (normalidade) e análise visual para os demais.

Foi realizada a análise de correlação linear de Pearson entre as propriedades avaliadas. Utilizou-se $\mathrm{o}$ teste $\mathrm{t}$ a $5 \%$ de significância para verificar se as correlações encontradas eram estatisticamente diferentes de zero.

A classificação do coeficiente de correlação (r) baseou-se no critério de Callegari- Jacques (2003) que prevê, em módulo, as seguintes condições: fraca correlação (baixa) para $0<\mathrm{r}<0,30$; moderada correlação para $0,30 \leq \mathrm{r}<0,60$; correlação alta (forte) para $0,60 \leq \mathrm{r}<0,9$; correlação muito forte para $r \geq 0,90$ e correlação perfeita para $r=1,00$.

\section{RESULTADOS E DISCUSSÃO}

Analisando a base de dados, observou-se que a maior energia específica de corte foi requisitada durante o processamento de uma amostra da espécie Hymenaea spp em que foi consumido $0,1664 \mathrm{KJ}$ para realizar o corte de $1 \mathrm{~cm}^{3}$ de madeira. O menor valor de energia específica de corte por amostra foi observado durante o corte da espécie T. ciliata $\left(0,0451 \mathrm{KJ}^{*} \mathrm{~cm}^{-3}\right)$. A diferença entre a maior e a menor energia específica requisitada foi de $73 \%$.

As médias da energia específica de corte e umidade, por espécie encontram-se na Tabela 2.

Tabela 2. Valores médios de umidade base seca e da energia específica para o corte transversal para as nove espécies avaliadas.

\begin{tabular}{lcc}
\hline \multicolumn{1}{c}{ Espécie } & UBS $(\%)$ & $\begin{array}{c}\text { Energia Específica de } \\
\text { Corte }\left(\mathrm{KJ}^{*} \mathrm{~cm}^{-3}\right)\end{array}$ \\
\hline T. ciliate & 16,6 & 0,0508 \\
C. japonica & 20,1 & 0,0667 \\
T. grandis & 16,3 & 0,0677 \\
E. grandis & 18,0 & 0,0753 \\
Q. dinizii & 15,9 & 0,0810 \\
E. saligna & 19,4 & 0,0907 \\
B. nitida & 13,0 & 0,1049 \\
Hymenaea spp & 14,8 & 0,1295 \\
T. serratifolia & 12,4 & 0,1068 \\
\hline
\end{tabular}

A análise de variância da energia específica de corte no sentido transversal da madeira para todas as espécies encontra-se na Tabela 3. Observa-se que houve diferença estatística entre as médias das espécies, a 5\% de significância. 
Tabela 3. Resumo da análise de variância da energia específica de corte para o corte transversal em serra circular de carrinho

\begin{tabular}{ccc}
\hline $\begin{array}{c}\text { Fonte de } \\
\text { Variação }\end{array}$ & Grau de Liberdade & Quadrado Médio \\
\hline Espécie & 8 & $0.00299089^{*}$ \\
Resíduo & 36 & 0.00024367 \\
\hline TOTAL & 44 & \\
\hline CV $(\%)$ & 18,16 & \\
\hline
\end{tabular}

*Significativo a $5 \%$ de significância; ${ }^{\text {n.s. }}$ não-significativo a $5 \%$ de significância

Por meio da comparação múltipla das médias pelo teste de Scott-Knott, a 5\% de significância (FIGURA 3), observou-se que a energia específica de corte foi separada em três classes distintas.

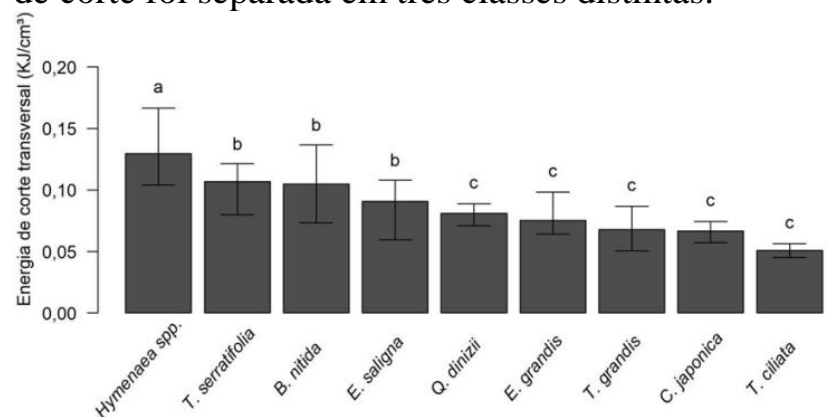

Médias seguidas de, pelo menos, uma mesma letra não diferem entre si a 5\% de significância, pelo teste de ScottKnott.

Figura 3 - Comparação múltipla das médias para o parâmetro energia específica para o corte transversal.

Andrade (2018) observou resultados semelhantes ao analisar o consumo de energia durante o fresamento periférico de em madeiras de espécies distintas. Dessa forma, corroborando com o trabalho em quem mostra que o material genético influencia na energia requerida para a realização do corte.

Observando os dados de umidade base seca notou-se que a amostra com maior umidade de equilíbrio, 20,6\%, foi da espécie C. joponica. A menor umidade de equilíbrio foi encontrada em amostra de $T$. serratifoilia, com umidade base seca de $12,1 \%$. O coeficiente de variação dos dados de umidade base seca foi de apenas $3,01 \%$ e isso pode ser explicado pois as tábuas estavam sujeitas a mesmas condições de armazenamento, estando todas em equilíbrio com o ambiente no momento do corte.

A análise de variância da umidade base seca da madeira para as nove espécies, encontra-se na Tabela 4. Observa-se que houve diferença estatística entre as médias das espécies, a 5\% de significância.

Tabela 4. Resumo da análise de variância da umidade base seca para as amostras das nove espécies avaliadas

\begin{tabular}{ccc}
\hline Fonte de & Grau de Liberdade & Quadrado Médio \\
Variação & 8 & $34.309^{*}$ \\
Espécie & 36 & 0,240 \\
Resíduo & 44 & \\
\hline TOTAL & 3,01 & \\
\hline CV $(\%)$ & & \\
\hline
\end{tabular}

*Significativo a 5\% de significância; ${ }^{\text {n.s. }}$ não-significativo a $5 \%$ de significância
Por meio da comparação múltipla das médias pelo teste de Scott-Knott, a 5\% de significância (FIGURA 4), observa-se que a umidade base seca foi separada em seis classes distintas.

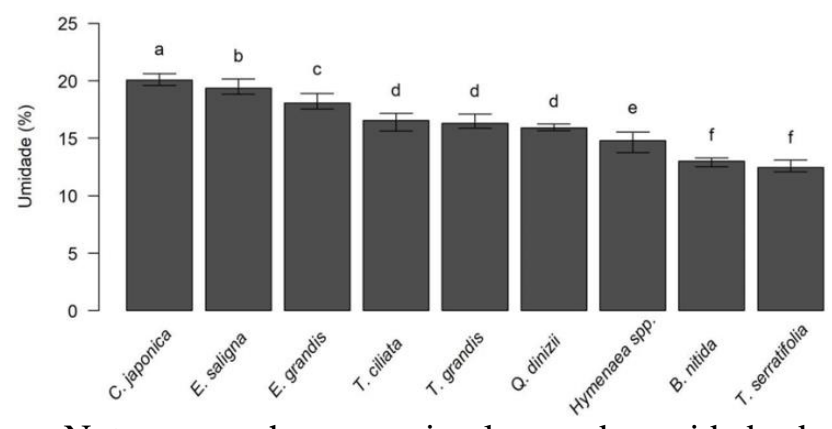

Nota-se que houve mais classes de umidade do que de energia específica de corte e isso pode ser relacionado ao comportamento individual das madeiras frente a umidade do ambiente, em que observa-se que algumas espécies são mais sensíveis a adsorção de água da atmosfera ambiente, sendo espécies mais ou menos higroscópicas do que outras.

A correlação entre a umidade base seca e a energia de corte para os cortes transversais encontrase na Figura 5.

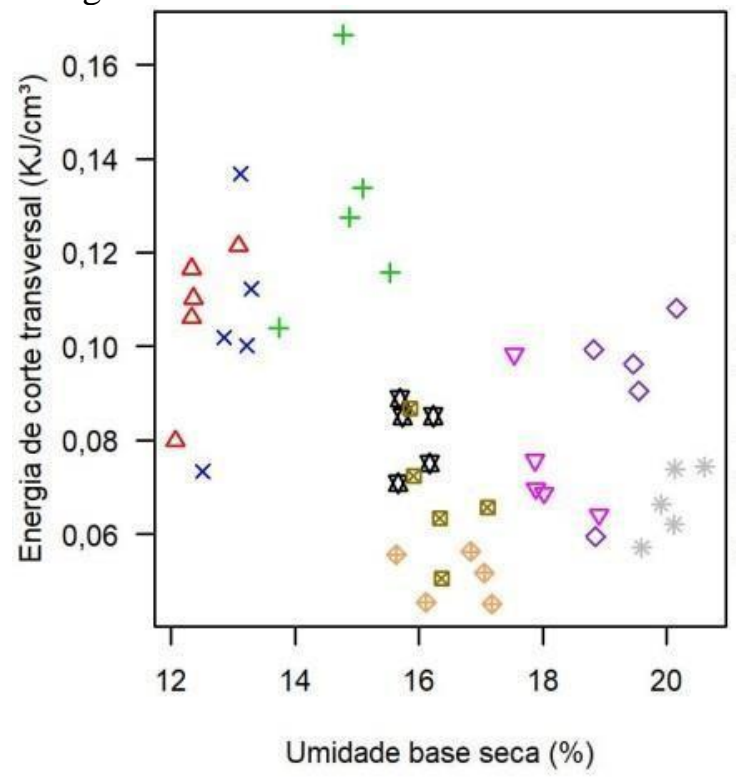

Figura 5 - Correlação de Pearson entre os dados de umidade base seca e de energia específica de corte para os cortes transversal para as nove espécies.

A relação entre energia específica requerida durante $\mathrm{o}$ processamento transversal em serra circular e a umidade base seca foi inversamente proporcional $(\mathrm{r}=-0,462)$ e foi considerada mediana. Observa-se que madeiras mais úmida requereram em média menores valores de energia específica de corte. Esses dados corroboram com estudo realizado por Guedes (2016) em que a autora observou influencia da umidade na energia requerida durante o fresamento periférico de três espécies sendo os 
cortes realizados com a madeira exposta a altas, médias e baixas umidades.

\section{CONCLUSÕES}

Conclui-se que a umidade exerce de fato influência no corte. A umidade afeta de forma inversa no quanto a madeira vai requerer de esforço durante seu processamento mecânico.

\section{REFERÊNCIAS}

ANDRADE, A. C. A., OLIVEIRA, M, B., SILVA, J. R. M., MOULIN, J. C., SOUZA, M.T., LIMA, L. C. Quality of machined surfaces and specific cutting energy in wood of two African mahogany species. Scientia Forestalis, volume 46, n. 120 Dezembro de 2018.

ANDRADE, A. C. de A. Investigação da energia de corte em madeiras de diferentes densidades básicas. 2018. 32p. Tese (Doutorado em Tecnologia da Madeira) Universidade Federal de Lavras, Lavras-MG.

ASSOCIAÇÃO BRASILEIRA DE NORMAS TÉCNICAS (ABNT). NBR 7190: Projetos de estruturas de madeira - Rio de Janeiro, 1997. 107p

BRAGA, P.P.C. Estabelecimento de padrões de usinagem e qualidade de acabamento em madeira de Coffea arabica. 2011. 57p. Dissertação (Mestrado em Tecnologia da Madeira) - Universidade Federal de Lavras, Lavras-MG.

CALLEGARI-JACQUES, S. M. Bioestatística: princípios e aplicações. Porto Alegre: Artmed, 2003. 255 p.

EYMA, F.; MEAUSOONE, P. J.; MARTIN, P. Strains and cutting forces involved in the solid wood rotating cutting process. Journal of Materials Processing Technology, v. 148, n. 2, p. 220-225, 2004.

FRANZ, N. C. An analysis of the wood-cutting process. Ann. Arbor: University of Michigan Press, 1958. 600p.
GUEDES, T.O. Consumo de energia específica de corte em madeiras de diferentes densidades em distintas umidades. 2016. 83p. Dissertação (Mestrado em Tecnologia da Madeira) - Universidade Federal de Lavras, Lavras-MG.

KOCH, P. Wood machining process. New York: Ronald press company, 1964. $530 \mathrm{p}$.

LOEHNERTZ, S. P.; COOZ, I. V. Sawtooth forces in cutting tropical hardwoods native to South America. Washington: US Forest Service, 1998. 16 p. (Research Paper FPL- RP, 567).

LUČIĆ, R. B.; GOGLIA, V.; PERVAN, S.; DUKIC, I.; RISOVIC, S. The influence of wood moisture content on the process of circular rip-sawing. Part I: Power requirements and specific cutting forces. Wood Research, Kyoto, v. 49, n. 1, p. 41-49, 2004.

MORADPOUR, P.; DOOSTHOSEINI, K.; SCHOLZ, F.; TARMIAN, A. Cutting forces in band saw processing of oak and beech wood as affected by wood moisture content and cutting directions. European Journal of Wood and Wood Products, HolzRoh Werkst, v. 71, n. 6, p. 747-754, 2013.

SCOTT, A. J.; KNOTT, M. A cluster analysis method for grouping means in the analysis of variance. Biometrics, Washington, US, v. 30, n. 2, p. 507-512, 1974. DOI: $10.2307 / 2529204$.

SOUZA, E. M. Influência dos parâmetros de usinagem da madeira de Eucalyptus sp. no consumo de energia específica de corte em serra circular. 2009. 50p. Dissertação (Mestrado em Ciência e Tecnologia de Madeira) - Universidade Federal de Lavras, Lavras, 2009. 